\title{
Ion Beam Nanodissection for 3D Compositional Mapping of Mammalian Cells
}

\author{
J. Fu*, K. Narayan*, T. Prosa** and S. Subramaniam* \\ * National Cancer Institute, National Institutes of Health, Bethesda, MD 20892 USA \\ ** Imago Scientific Instruments Corp., 5500 Nobel Drive, Madison, WI 53711-4951 USA
}

Localizing atoms and molecules in cells or tissues is of great importance for biological studies, and new strategies, such as EELS and SIMS, are being developed for subcellular level mapping of compositional and chemical distribution. The maximum volume that can be mapped accurately is often limiting, and thus biological specimens are frequently sectioned to extend high resolution to more dimensions. However, conventional microtome-based sectioning of biological specimens has a number of limitations which have been addressed by Ion Abrasion SEM (IA-SEM), an emerging strategy for imaging three-dimensional ultrastructures [1]. With Focused Ion Beam (FIB) milling to remove thin slices from a bulk sample, SEM imaging can be performed to visualize cellular architecture and biological structures through reconstruction [1-3]. The ability of the FIB to abrade away areas of pre-determined thickness in specific regions of the specimen can be exploited to reveal 3D structures of complex biological organelles. These newly exposed surfaces are now amenable to high-resolution but low-penetration techniques such as SIMS.

FIB protocols can be modified and extended to the tissue level as well. Although laser based dissection methods, such as Laser Capture Microdissection (LCM), have been developed to extract site-specific sections from tissue to facilitate various bioanalyses [4], dividing a single cell into arbitrary geometries in a timely fashion, without compromising the integrity of molecular arrangements, still remains a challenge. We demonstrate here that FIB can be used as an effective solution for sectioning freeze-dried, unfixed cells and tissues for downstream analysis. $30 \mathrm{keV} \mathrm{Ga}$ based FIB milling, with a typical beam spot size below $100 \mathrm{~nm}$, is performed to create predefined micrometer or submicrometer geometries on target cells (FIG. 1). Site-specific intracellular regions from single cells can be individually separated and transferred, and thus become independently accessible for a wide range of detection methods.

Nanoscale dissection of mammalian cells by FIB and the accompanying sample preparation protocols provide an approach to divide cells at unprecedented resolution with minimal physical and chemical changes. By removing spatial limitations that have long impeded single cell analysis, molecular detection and identification are now only limited by the sensitivity of analytical tools. FIG. 2 shows representative images from Atom Probe Tomography (APT) experiments. Spatial mapping of individual atoms by APT is highly dependent on the ability to shape the sample into sharp needle-like entities ( $\sim 100 \mathrm{~nm}$ diameter). Applying these novel sample preparation and FIB protocols, we now present the spatial atomic map of a small subcellular volume of a human (HeLa) cell in a near native state.

\section{References}

[1] J.A. Heymann et al., J. Struct. Biol. 155 (2006) 63

[2] J.A. Heymann et al, J. Struct. Biol. 166 (2009) 1

[3] A.E. Bennett et al., PLoS Pathog. (2009) 
[4] M.R. Emmert-Buck et al., Science. 274 (1996) 998

[5] T.J. Prosa et al., Microsc. Microanal. 16 (Suppl. 2) (2010) submitted
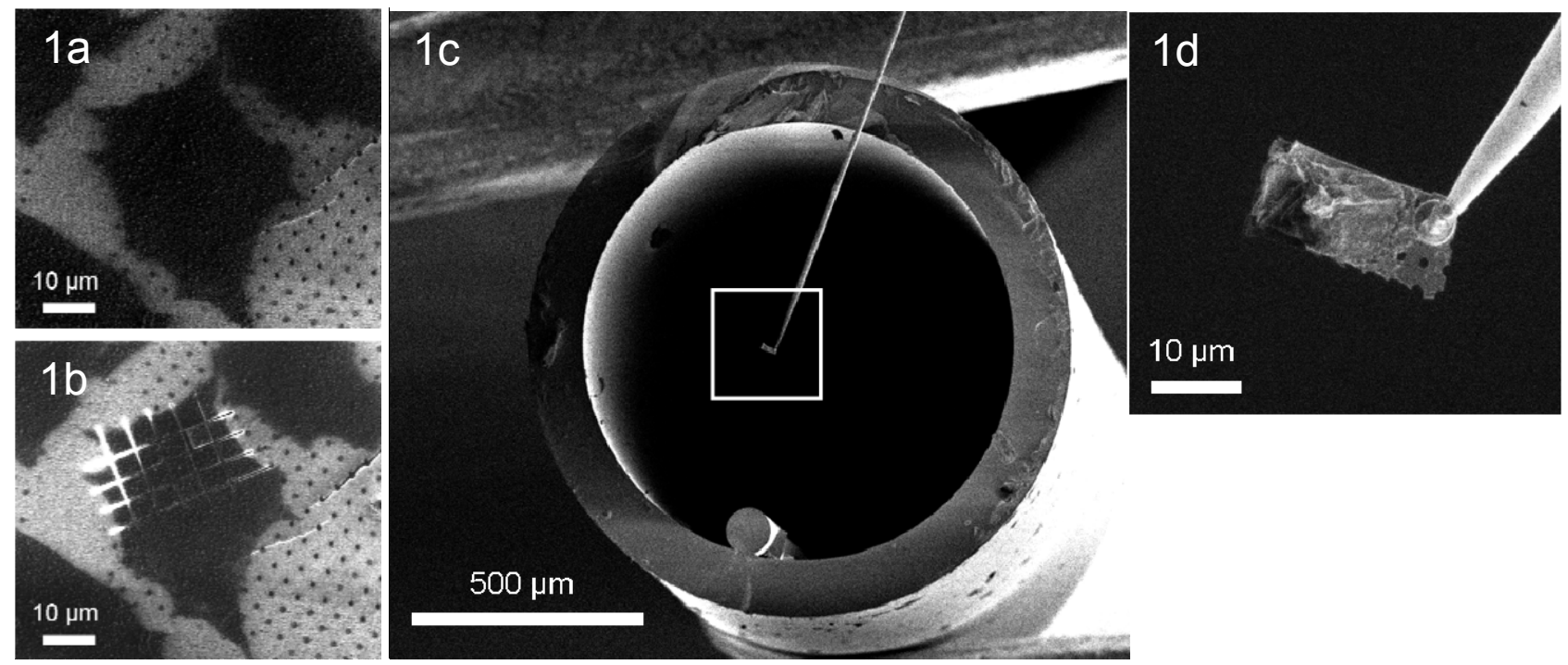

FIG. 1. SEM images of subcellular portion of a frozen cell on carbon film (a) before and (b) after dissection by FIB at $100 \mathrm{~K}$ (c) A subcellular freeze dried dissect on film was lifted out by a tungsten probe and transferred to the end of a standard $2 \mathrm{~mm}$ glass pipette. (d) Enlarged image of the cellular dissect (e) FIB dissection on dried mouse liver tissue on a glass slide with gold coating.

FIG. 2. Nanoscale chemical mapping on intracellular structure of freeze dried cells. (a) SEM image of subcellular dissect by FIB milling to form a needle shape and (b) nanometer resolution compositional map by APT analysis showing selected ions: m/z 12 (carbon) in green, m/z 14 (nitrogen) in blue and $\mathrm{m} / \mathrm{z} 69$ (implanted gallium) in red.
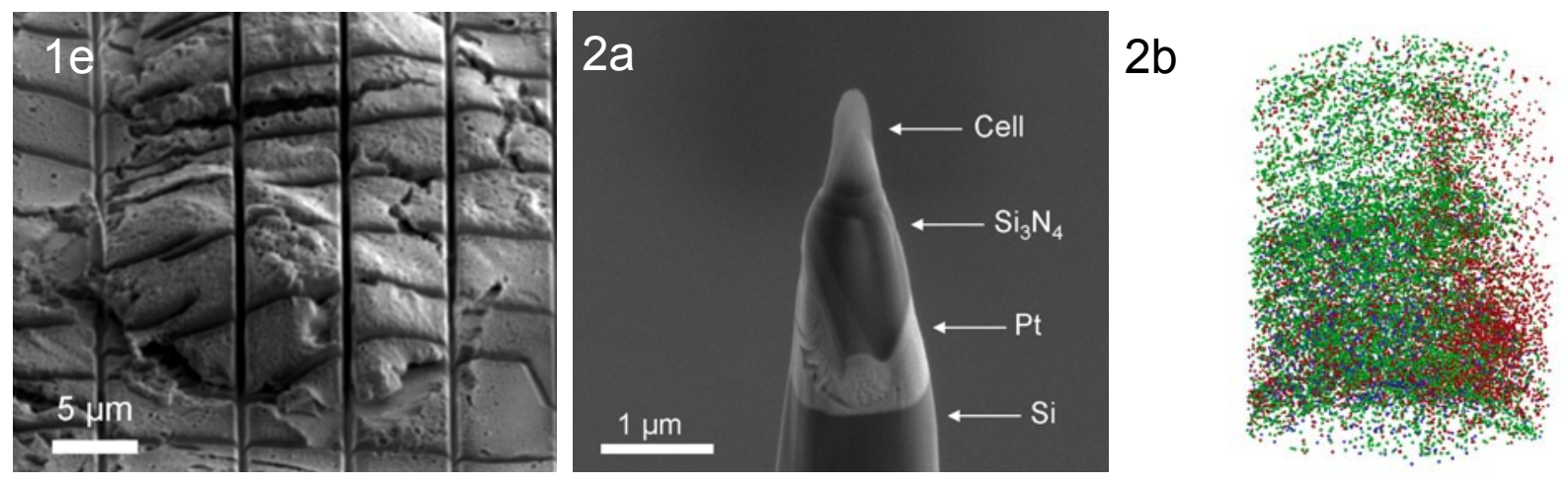